\title{
A medicina veterinária na saúde pública: abordagem em saúde única diante da pandemia do covid-19
}

\begin{abstract}
A saúde pública, como segmento da ciência veterinária provoca uma reflexão acerca da integração das áreas profissionais da saúde. A essa abordagem se dá o nome de Saúde Única, que vem ganhando espaço nos países do mundo, com objetivo de promoção de saúde. A pandemia da COVID-19 vem sendo uma promotora das adaptações da ciência e das políticas públicas, parecendo evidenciar pontos de falha no sistema de saúde tal como atualmente constituído e estruturado. Nesse sentido, indaga-se se uma maior participação da medicina veterinária, enquanto componente da saúde pública, poderia contribuir com a mitigação dos efeitos da COVID-19. Por intermédio de uma pesquisa exploratória, traça-se uma revisão bibliográfica para fortalecer a discussão teórica. Foi realizada uma pesquisa nos Descritores em Ciência da Saúde (DeSC); seguida de levantamento bibliográfico em plataformas de dados: ScienceDirect, BVS (Biblioteca Virtual em Saúde), Periódicos Capes e informações complementares no Conselho Federal de Medicina Veterinária do Brasil e no Center for Disease Control and Prevention dos Estados Unidos. A busca deu-se por artigos científicos indexados nos últimos 10 anos, considerando-se toda a base de dados. Foram selecionados para o desenvolvimento desta pesquisa artigos acadêmicos nos idiomas português e inglês, sendo encontrados 101 artigos de revisão, originais, e relatos de casos nos idiomas inglês e português. Na segunda etapa, dentre os resumos levantados, foi feita uma seleção daqueles que contemplavam ao menos 2 palavras-chave e cuja formulação fosse igual ou aproximada à proposta de busca realizada para este estudo, seguindo-se de análise rigorosa de conteúdo para verificar a adesão à temática proposta. Como resultado, vislumbra-se que a utilização do sistema estruturado na ideia da saúde única, diferentes impactos com relação à pandemia poderiam ter sido obtidos.
\end{abstract}

Palavras-chave: Saúde Única; COVID-19; Medicina Veterinária.

\section{Veterinary medicine in the public health: approach in one health of the pandemic of covid-19}

Public health, as a segment of veterinary science, provokes a reflection on the integration of professional health sectors. This approach is called One Health, which has got stronger in countries around the world. The COVID-19 pandemic has been a promoter of the adaptations of science and public policies, seeming to show points of failure in the health system as it is currently constituted and structured. In this sense, it is asked whether greater participation of veterinary medicine, as a component of public health, could contribute to mitigating the effects of COVID-19. Through exploratory research, a bibliographic review is designed to strengthen the theoretical discussion. The research was carried out on Descritores em Ciência da Saúde (DeSC); followed by a bibliographic survey on data platforms: ScienceDirect, BVS (Biblioteca Virtual em Saúde), Periódicos Capes and complementary information at the Federal Council of Veterinary Medicine of Brazil and the Center for Disease Control and Prevention in the United States. A search for indexed scientific articles was carried out in the last 10 years, considering the database. Academic articles in Portuguese and English were selected for the development of this research, counting 101 original review articles and case reports in English and Portuguese. In the second stage, among the abstracts raised, a selection was made of those that included at least 2 keywords and whose formulation was equal to or similar to the research proposal carried out for this study, followed by rigorous content analysis to verify adherence to the proposed theme. As a result, it is envisaged that using the structured system in the idea of One Health, different impacts concerning the pandemic could have been obtained.

Keywords: One Health; COVID-19; Veterinary Medicine.

Topic: Ciências Sociais e Humanas em Saúde

Reviewed anonymously in the process of blind peer.

Ricardo Vinícius Duarte Cavalcanti (iD)

Centro Universitário Brasileiro, Brasil http://lattes.cnpq.br/7696479967138260

http://orcid.org/0000-0002-7580-894X

ricardoviniciusd@gmail.com
Received: $\mathbf{1 7} / \mathbf{1 0} / \mathbf{2 0 2 0}$

Approved: 21/01/2021
Referencing this:

CAVALCANTI, R. V. D.. A medicina veterinária na saúde pública: abordagem em saúde única diante da pandemia do covid-19. Scire Salutis, v.11, n.1, p.127-133, 2021. DOI:

http://doi.org/10.6008/CBPC2236-9600.2021.001.0014 


\section{INTRODUÇÃO}

A história das pandemias mundiais trouxe aprendizados sobre melhores respostas e políticas para prevenção de emergências em saúde. A saúde pública, como segmento de estudo, está inserida diretamente nessa organização política e social, tendo o médico veterinário uma atribuição de destaque para resolução de diretrizes nesses interesses.

Em dezembro de 2019, ocorreu um conjunto de casos de pneumonia, causado por um coronavírus recém identificado em Wuhan, na China. Estudos sugerem que morcegos podem ter sido o potencial reservatório da síndrome respiratória aguda grave coronavírus 2 (SARS-CoV-2), mostrando que a composição genética do novo coronavírus é $96 \%$ idêntica à encontrada em morcegos e que a doença parece ter se originado em um mercado de frutos do mar naquela localidade, onde animais selvagens, incluindo marmotas, morcegos e cobras são comercializados ilegalmente (ZHOU et al., 2020). Sabendo-se que o vírus foi transmitido a partir de animais para humanos, acredita-se que os primeiros casos foram de pessoas que frequentavam o local e tiveram contato com os animais (ZHOU et al., 2020). Os sintomas mais comuns relatados foram: febre, tosse e dificuldade em respirar (MACEDO JÚNIOR, 2020).

Diante da problemática trazida pela pandemia nos mais diversos setores, o sistema de saúde tal como compreendido atualmente parece evidenciar uma falha estrutural. Por isso, é salutar investigar de que maneira a abordagem por meio do conceito de Saúde Única (One Health) pode provocar uma releitura do protagonismo entre as diferentes ciências que compõem o segmento, evidenciando pontos de contribuição da medicina veterinária nesse contexto.

O artigo parte do tema da saúde pública e sua intersecção com a medicina veterinária, aliando os conhecimentos produzidos em relação às zoonoses para fazer inferências sobre o questionamento principal. A hipótese, nesse sentido, parte da premissa de que pode ser estabelecida uma colaboração e afinação entre os papéis das ciências da saúde no sentido de mitigar impactos pandêmicos, tomando-se como base o caso do COVID-19.

Assim, visa-se analisar, à luz do conceito de saúde única, as contribuições palpáveis da veterinária no cenário pandêmico. Para tanto, faz-se uma breve análise da saúde pública, histórico, conceito, direcionando às zoonoses e as contribuições da veterinária normalmente verificadas nesses casos; em seguida, avalia-se o conceito da saúde única; aprofunda a situação da COVID-19; e, aponta-se, com o apoio da literatura especializada, pontos de destaque na junção dos conceitos trabalhados, centrando-se na atual hipótese.

\section{METODOLOGIA}

Este trabalho foi desenvolvido por meio de uma revisão bibliográfica integrativa. Para determinação das palavras-chave e para a busca de artigos científicos nas bases de dados, foi realizada uma pesquisa nos Descritores em Ciência da Saúde. Os seguintes termos foram utilizados: Saúde única, medicina veterinária e coronavírus. A segunda etapa, realizou-se o levantamento bibliográfico nas seguintes plataformas de dados: ScienceDirect, BVS (Biblioteca Virtual em Saúde), Periódicos Capes e informações complementares no 
Conselho Federal de Medicina Veterinária do Brasil e no Center for Disease Control and Prevention dos Estados Unidos. Foi realizada busca de artigos científicos indexados nos últimos 10 anos, considerando-se toda a base de dados. Foram selecionados para o desenvolvimento desta pesquisa artigos acadêmicos nos idiomas português e inglês, sendo encontrados 101 artigos de revisão, originais, e relatos de casos nos idiomas inglês e português. Na segunda etapa, dentre os resumos levantados, foi feita uma seleção daqueles que contemplavam ao menos 2 palavras-chave e cuja formulação fosse igual ou aproximada à proposta de busca realizada para este estudo. Em seguida, foram submetidas à análise rigorosa de conteúdo para verificar a adesão à temática proposta.

Tabela 1: Obras selecionadas.

\begin{tabular}{|c|c|c|c|}
\hline AUTOR & TíTULO (ANO) & $\begin{array}{l}\text { PLATAFORMA/BASE DE } \\
\text { DADOS }\end{array}$ & ASSUNTO \\
\hline AMUASI et al. & $\begin{array}{l}\text { Calling for a COVID-19 } \\
\text { One Health Research } \\
\text { Coalition (2020) }\end{array}$ & ScienceDirect & $\begin{array}{l}\text { Advoga-se pelo estabelecimento de uma } \\
\text { pesquisa em Saúde Única a respeito da } \\
\text { COVID-19, em uma aliança inclusiva para } \\
\text { fortalecer os vínculos com a mudança } \\
\text { climática e a comunidade de pesquisa em } \\
\text { saúde planetária. }\end{array}$ \\
\hline GOMES & $\begin{array}{l}\text { Importância e atribuições } \\
\text { do médico veterinário na } \\
\text { saúde coletiva (2017) }\end{array}$ & Periódicos Capes & $\begin{array}{l}\text { Defende-se que o médico veterinário tem } \\
\text { a responsabilidade de proporcionar } \\
\text { melhores condições ambientais, difusão } \\
\text { de informações e orientação à população } \\
\text { humana quanto aos princípios básicos de } \\
\text { saúde, sobretudo no contexto de Saúde } \\
\text { Única. }\end{array}$ \\
\hline $\begin{array}{l}\text { CENTER FOR DISEASE } \\
\text { CONTROL AND } \\
\text { PREVENTION (CDC) }\end{array}$ & $\begin{array}{l}\text { Connecting human, } \\
\text { animal, and } \\
\text { environmental health } \\
(2020)\end{array}$ & $\begin{array}{l}\text { National Center for } \\
\text { Emerging and Zoonotic } \\
\text { Infectious Diseases }\end{array}$ & $\begin{array}{l}\text { Reconhece-se a conexão entre a saúde das } \\
\text { pessoas e a saúde dos animais e ambiente } \\
\text { compartilhado. Defesa da abordagem em } \\
\text { Saúde Única. }\end{array}$ \\
\hline $\begin{array}{l}\text { CONSELHO FEDERAL DE } \\
\text { MEDICINA VETERINÁRIA }\end{array}$ & $\begin{array}{l}\text { O que é saúde única? } \\
\text { (2015) }\end{array}$ & $\begin{array}{l}\text { CONSELHO FEDERAL DE } \\
\text { MEDICINA VETERINÁRIA }\end{array}$ & $\begin{array}{l}\text { Campanha destinada a elucidar a atuação } \\
\text { do médico veterinário. Evidencia-se a } \\
\text { presença do profissional referido em } \\
\text { diversos aspectos do cotidiano da } \\
\text { população. Argumenta-se pelo papel de } \\
\text { impacto do veterinário na saúde global. }\end{array}$ \\
\hline DAVIS & $\begin{array}{l}\text { Rethinking One Health: } \\
\text { emergent human, animal } \\
\text { and environmental } \\
\text { assemblages (2020) }\end{array}$ & ScienceDirect & $\begin{array}{l}\text { Defende o crescimento das perspectivas } \\
\text { em Saúde Única e a influência na saúde } \\
\text { global. Apresenta-se a Saúde Única como } \\
\text { inerentemente interdisciplinar e } \\
\text { integradora, reunindo a saúde humana, } \\
\text { animal e ambiental. }\end{array}$ \\
\hline DECARO & $\begin{array}{l}\text { COVID-19 from veterinary } \\
\text { medicine and one health } \\
\text { perspectives: what animal } \\
\text { coronaviruses have } \\
\text { taught us (2020) }\end{array}$ & ScienceDirect & $\begin{array}{l}\text { Apoia a formulação de políticas públicas } \\
\text { com o apoio da medicina veterinária. } \\
\text { Sustenta um sistema sólido e sustentável } \\
\text { de medidas para a gestão do meio } \\
\text { ambiente e dos animais. Defende-se, } \\
\text { assim, o avanço do movimento global } \\
\text { "One Health". }\end{array}$ \\
\hline JACOBSEN & $\begin{array}{l}\text { Will COVID-19 generate } \\
\text { global preparedness? } \\
\text { (2020) }\end{array}$ & $\begin{array}{l}\text { Biblioteca Virtual em } \\
\text { Saúde (BVS) }\end{array}$ & $\begin{array}{l}\text { Alude para o surto de COVID-19 a } \\
\text { necessidade de compromisso intensificado } \\
\text { e sustentado para preparação da saúde } \\
\text { pública global. }\end{array}$ \\
\hline MACEDO JÚNIOR & $\begin{array}{l}\text { COVID-19: calamidade } \\
\text { pública (2020) }\end{array}$ & $\begin{array}{l}\text { Biblioteca Virtual em } \\
\text { Saúde (BVS) }\end{array}$ & $\begin{array}{l}\text { Identifica-se questões importantes } \\
\text { relacionadas a pandemia do Covid-19. }\end{array}$ \\
\hline $\begin{array}{l}\text { ORGANIZAÇÃO PAN- } \\
\text { AMERICANA DA SAÚDE }\end{array}$ & $\begin{array}{l}\text { Guia para a aplicação do } \\
\text { instrumento de medição } \\
\text { do desempenho das } \\
\text { funções essenciais de } \\
\text { saúde pública (2010) }\end{array}$ & OPAS & $\begin{array}{l}\text { Fornece-se uma visão geral da saúde } \\
\text { pública e das funções essenciais da saúde } \\
\text { pública } \\
\text { nas Américas. }\end{array}$ \\
\hline PFUETZENREITER et al. & Evolução histórica da & Periódicos Capes & Aborda-se a evolução histórica da \\
\hline
\end{tabular}




\begin{tabular}{|l|l|l|l|}
\hline & $\begin{array}{l}\text { medicina veterinária } \\
\text { preventiva e saúde } \\
\text { pública (2014) }\end{array}$ & $\begin{array}{l}\text { participação da medicina veterinária na } \\
\text { saúde pública e as ações desempenhadas } \\
\text { pelo médico veterinário. }\end{array}$ \\
\hline $\begin{array}{l}\text { VETERINARIANS } \\
\text { WITHOUT BORDERS } \\
\text { (VWB) }\end{array}$ & $\begin{array}{l}\text { One Health for one world: } \\
\text { a compendium of case } \\
\text { studies (2020) }\end{array}$ & VWB & $\begin{array}{l}\text { Relata-se algumas das iniciativas em Saúde } \\
\text { Única que incentivaram uma jornada em } \\
\text { direção a uma compreensão mais } \\
\text { integrativa de saúde e bem-estar. }\end{array}$ \\
\hline ZHOU et al. & $\begin{array}{l}\text { A pneumonia outbreak } \\
\text { associated with a new } \\
\text { coronavirus of probable } \\
\text { bat origin (2020) }\end{array}$ & ScienceDirect & $\begin{array}{l}\text { Identifica-se e caracteriza-se o novo } \\
\text { coronavírus (2019-nCoV), que causou uma } \\
\text { epidemia de síndrome respiratória aguda } \\
\text { em humanos em Wuhan, China. }\end{array}$ \\
\hline HUESTON & $\begin{array}{l}\text { Veterinary medicine: } \\
\text { public good, private good } \\
\text { or both? (2016) }\end{array}$ & $\begin{array}{l}\text { Biblioteca Virtual em } \\
\text { Saúde (BVS) }\end{array}$ & $\begin{array}{l}\text { Destá à beira de uma nova era de relevância } \\
\text { e importância para bens públicos e } \\
\text { privados. }\end{array}$ \\
\hline
\end{tabular}

DISCUSSÃO TEÓRICA

\section{Medicina Veterinária na Saúde Pública}

De acordo com a Organização Mundial da Saúde (OMS), Saúde pública se refere a todas as medidas organizadas (públicas ou privadas) para prevenir doenças, promover saúde e prolongar a vida da população como um todo. Apesar dessa atual atribuição, a saúde nem sempre foi pensada como algo público, somente com a expansão das cidades, o acréscimo de pessoas ocupando os mesmos espaços, é que as populações passaram a ser julgadas como algo a ser preservado, fenômeno que ocorreu a partir do século XVII na Europa.

No que concerne à iniciativa de saúde pública nas américas, é relevante destacar dentro das Funções Essenciais de Saúde Pública (FESP), definidas pela Organização Pan-Americana de Saúde (OPAS), a de número onze, que compreende "a redução do impacto de emergências e desastres em saúde" (2010).

Nesse sentido, a OMS reconhece a participação do médico veterinário no planejamento e avaliação das medidas preventivas e de controle adotadas pelas equipes de Saúde Pública (GOMES, 2017). Isso porque, desde a formação acadêmica, os profissionais da veterinária estão em constante contato com o tronco comum de disciplinas básicas em saúde e, durante a experiência prático-profissional, podem desempenhar ações, dentre as quais, abstratamente: atividades estratégicas de vigilância, prevenção e controle de zoonoses de relevância para a saúde pública.

No que tange às zoonoses, em específico, a medicina veterinária pode exercer a função de ponte, tanto pela atenção à saúde animal, quanto à saúde ambiental. Exemplo disso são as medidas de vigilância de alimentos que, embora não estejam diretamente ligadas a um agente infeccioso, reforçam o papel da veterinária na prevenção de doenças. Além disso, nota-se que a referida ciência tem se voltado a ações coletivas, auxiliando na instauração de agendas como: a quarentena, o sacrifício de animais enfermos (PFUETZENREITER et al., 2004), bem como os trabalhos educacionais voltados à prevenção e vigilância de zoonoses locais como, por exemplo, no Brasil, a criação do Núcleo de Apoio à Família (NASF) — que tem a participação de um corpo de médicos veterinários.

\section{A Importância da Saúde Única}

Atualmente, 13 zoonoses são responsáveis pela marca de 2,5 bilhões de doenças humanas, que 
resultam em 2,7 milhões de mortes por ano (CDC, 2020). Dessa forma, governos e cientistas reconheceram que a colaboração interdisciplinar é necessária para prevenir e controlar estas doenças. Percebe-se, então, que o conceito de saúde única (One Health) definido pela OMS, abrange uma visão integrada, que considera a indissociabilidade entre saúde humana, animal e ambiental, principalmente no contexto das políticas de prevenção.

A Saúde Única define políticas, legislações, pesquisa e implementação de programas, em que múltiplos setores se comunicam e trabalham em conjunto nas ações para a diminuição de riscos e manutenção da saúde (CFMV, 2015). Consequentemente, essa integração pode contribuir para a eficácia das ações em Saúde Pública, com redução dos riscos para a saúde global (CFMV, 2015). Tal questão pode ser observada na raiva humana, doença que consegue ser efetivamente prevenida somente mirando no animal como fonte do vírus (por exemplo, na vacinação de cães) (DAVIS et al., 2020).

Em Madagascar, a abordagem em saúde única ajudou a diminuir o número de casos da "febre do Vale do Rift" durante o surto de 2008, através de uma abordagem integrada entre os Ministérios locais da Saúde e da Agricultura, a Organização das Nações Unidas para Alimentação e Agricultura e a Organização Mundial da Saúde Animal, auxiliando na previsão e mapeamento do surto (VWB, 2010). Nota-se, portanto, que essas conjunções transdisciplinares e multisetoriais, como consideram Jacobsen et al. (2020), são necessárias na resolução de problemas complexos que ameaçam a saúde e segurança pública global, mais especificamente em relação a doenças como o COVID-19, porque a saúde única foca no perigo existente entre a relação humana e a vida selvagem. Nesse sentido, acrescentam os referidos autores que, a habilidade de detectar novas doenças em animais de pecuária e em animais selvagens são elementos cruciais e servem como alertas a pandemias humanas (JACOBSEN et al., 2020).

\section{Contexto da Difusão do SARS-COV-2}

Ao contrário das epidemias do SARS (com epicentro na Ásia entre 2002 e 2003) e MERS (ocorrida majoritariamente no Oriente médio em 2012), a pandemia do SARS-CoV-2 tem como característica uma baixa taxa de letalidade e uma grande quantidade de portadores assintomáticos, o que favoreceu a rápida propagação, principalmente em virtude da globalização e do encurtamento de distâncias promovido pela malha aérea. Após a China, o outro grande país a ser atingido foi a Itália, principiando, então, o imenso impacto mundial que se instaurou pela Europa e, sucessivamente, pelos demais continentes.

A pandemia declarada pela OMS em 11 de março, já resultou em mais de 600 mil mortes $^{1}$ de acordo com a Universidade Americana Johns Hopkins, e trouxe consigo diversos impactos econômicos e sociais como o fechamento de escolas, fronteiras e empresas, levando grande parte da população mundial a aderir a medidas isolamento social (quarentena, lockdown). Todos esses esforços com a finalidade de reduzir a propagação do vírus.

\footnotetext{
${ }^{1}$ Marco atingido durante a edição deste artigo (19 jul. 2020). Consultar: UNIVERSITY OF JOHNS HOPKINS. COVID-19 map. Disponível em: https://coronavirus.jhu.edu/map.html
} 
Destaca-se que a doença aqui comentada foi possivelmente transmitida ao ser humano através de uma cadeia de animais silvestres, como já explicitado, o que deve servir de base para o reforço da hipótese traçada no presente artigo, que contempla uma releitura de como a ciência é mobilizada no sentido da integração entre as diferentes especialidades da área da saúde.

\section{Pontos de Destaque Entre a Saúde Única: O Papel da Medicina Veterinária e a Pandemia do Covid-19}

Como afirma Decaro et al. (2020), ao comentar sobre os coronavírus animais, a medicina veterinária poderia ajudar no entendimento, origem e propagação do SARS-CoV-2, além de direcionar a medicina humana no desenvolvimento de vacinas.

Diante das circunstâncias, pode-se ter em mente o quão valiosa a veterinária pode ser para a criação de políticas públicas e gerenciamento do meio ambiente, dos animais e avanço do movimento global em saúde única (DECARO et al., 2020). Dessa mesma maneira, como afirma a VWB, "informações sobre a presente e antigas pandemias podem gerar ideias para um futuro planejamento, em particular comunicações diretas entre as diferentes áreas e cooperação entre a medicina humana e animal para uma melhor administração e prévia detecção de possíveis emergências" (VWB, 2010).

A Covid-19 desperta para a razão de ser do conceito de saúde única que, compartilhando de uma conexão intersetorial, se presta a assegurar a saúde e sustentabilidade futura do planeta (AMUASI et al., 2020). Isso porque, como afirma Hueston (2016), a ciência e a prática veterinária estão em uma "era de relevância e importância para bens públicos e privados", sugerindo uma "liderança compartilhada" entre os profissionais da saúde (HOUESTON, 2016).

\section{CONCLUSÕES}

Compreende-se, então, que a saúde pública possui como função básica a redução de impactos relacionados à saúde. Além disso, a inclusão do médico veterinário, se mostra essencial na definição de medidas de prevenção e controle de zoonoses de relevância para saúde.

Com a atual pandemia, a complexa relação existente entre pessoas, animais e meio ambiente ficou em maior evidência, sobretudo devido à instabilidade econômica advinda desta situação.

Por meio da efetivação de uma perspectiva de saúde única, a veterinária reveste-se de destaque na atuação e resolução de problemas locais e ou globais que ameaçam a saúde pública, agregando de maneira singular os conhecimentos animal e ambiental. Dessa maneira, fica evidente que, se mais países utilizassem de uma abordagem da saúde num contexto único, o impacto do COVID-19 poderia ter sido alterado.

Outro benefício, em si, se daria pelo próprio compartilhamento de conhecimentos entre as classes médicas humana e veterinária, trazendo vantagens no combate deste e de outros agentes infecciosos, auxiliando no manejo de doenças.

É esperado que a abordagem multidisciplinar em saúde única entrará em maior uso, podendo o veterinário fazer parte de equipes multiprofissionais para delimitação de estratégias efetivas e para prevenção de futuros surtos como o do COVID-19. Exemplo disso ocorre em países como os Estados Unidos, 
que possuem um organismo chamado 'CDC's One Health Office', escritório federal dedicado integralmente ao desenvolvimento de estratégias em saúde única, cuja sua diretora, Casey Barton, é uma médica veterinária.

\section{REFERÊNCIAS}

AMUASI, J. H.; WALZER, C.; HEYMANN, D.; CARABIN, H.; HUONG, L. T.; HAINES, A.; WINKLER, A. S.. Calling for a COVID-19 One Health Research Coalition. Lancet., v.395, n.10236, p.1543-1544, 2020. DOI: https://doi.org/10.1016/S0140-6736(20)31028-X

CDCP. Center for Disease Control and Prevention. Connecting human, animal, and environmental health. Fort Collins: National Center for Emerging and Zoonotic Infectious Diseases (NCEZID), 2020.

CFMV. Conselho Federal de Medicina Veterinária. O que é saúde única?. Brasília: CFMV, 2015.

DAVIS, A.; SHARP, J.. Rethinking One Health: emergent human, animal and environmental assemblages. Social Science \& Medicine, v.258, p.113093, 2020. DOI: https://doi.org/10.1016/j.socscimed.2020.113093

DECARO, N.; MARTELLA, V.; SAIF, L. J.; BUONAVOGLIA, C.. COVID-19 from veterinary medicine and one health perspectives: what animal coronaviruses have taught us. Res. Vet. Sci., v.131, p.21-23, 2020. DOI: https://doi.org/10.1016/j.rvsc.2020.04.009

GOMES, L. B.. Importância e atribuições do médico veterinário na saúde coletiva. Sinapse Múltipla, v.6, n.1, p.70-75, 2017.

HUESTON, W.. Veterinary medicine: public good, private good or both?. Veterinary Record, v.178, n.4, p.98-99, 2016.
JACOBSEN, K. H.. Will COVID-19 generate global preparedness?. Lancet, v.395, p.1013, 2020.

MACEDO JÚNIOR, A. M.. COVID-19: calamidade pública. Medicus, v.2, n.1, p.1-6, 2020. DOI:

http://doi.org/10.6008/CBPC2674-6484.2020.001.0001

OPAS. Organização Pan-Americana da Saúde. Guia para a aplicação do instrumento de medição do desempenho das funções essenciais de saúde pública. Washington: OMS, 2010.

PFUETZENREITER, M. R.; ZYLBEFFSZTAJN, A.; PIRES, F. D. A.. Evolução histórica da medicina veterinária preventiva e saúde pública. Ciência Rural, Santa Maria, v.34, n.5, p.16611668, 2004. DOI: https://doi.org/10.1590/S0103$\underline{84782004000500055}$

VWB. Veterinarians Without Borders. One Health for one world: a compendium of case studies. Ottawa: VWB, 2010.

ZHOU, P.; YANG, X.-L.; WANG, X.-G.; HU, B.; ZHANG, L.; ZHANG, W.; SI, H.-R.; ZHU, Y.; LI, B.; HUANG, C.-L.; CHEN, H.D.; CHEN, J.; LUO, Y.; GUO, H.; JIANG, R.-D.; LIU, M.-Q.; CHEN, Y.; SHEN, X.-R.; WANG, X.; ZHENG, X.-S.; ZHAO, K.; CHEN, Q.-J.; DENG, F.; LIU, L.-L.; YAN, B.; ZHAN, F.-X.; WANG, Y.-Y.; XIAO, G.-F.; SHI, Z.-L.. A pneumonia outbreak associated with a new coronavirus of probable bat origin. Nature, v.579, p.270-273, 2020. DOI: https://doi.org/10.1038/s41586-020-2012-7

A CBPC - Companhia Brasileira de Produção Científica (CNPJ: 11.221.422/0001-03) detém os direitos materiais desta publicação. Os direitos referem-se à publicação do trabalho em qualquer parte do mundo, incluindo os direitos às renovações, expansões e disseminações da contribuição, bem como outros direitos subsidiários. Todos os trabalhos publicados eletronicamente poderão posteriormente ser publicados em coletâneas impressas sob coordenação da Sustenere Publishing, da Companhia Brasileira de Produção Científica e seus parceiros autorizados. Os (as) autores (as) preservam os direitos autorais, mas não têm permissão para a publicação da contribuição em outro meio, impresso ou digital, em português ou em tradução. 